\title{
THE EXCEPTIONAL SUBSET OF A $C_{0}$-CONTRACTION
}

BY

\section{DOMINGO A. HERRERO}

ABSTRACT. Let $T$ be a $C_{0}$-operator acting on a (complex separable) Hilbert space $K$; i.e., $T$ is a contraction on $K$ and it satisfies the equation $q(T)=0$ for some inner function $q$, where $q(T)$ is defined in the sense of the functional calculus of B.Sz.-Nagy and C. Foias.. Among all those inner functions $q$ there exists a unique minimal function $p$ defined by the conditions: (1) $p(T)=0$; (2) if $q(T)=0$, then $p$ divides $q$. A vector $F \in \mathbb{K}$ is called exceptional if there exists an inner function $r$ such that $r(T) F=0$, but $p$ does not divide $r$. The existence of nonexceptional vectors plays a very important role in the theory of $C_{0}$-operators. The main result of this paper says that nonexceptional vectors actually exist; moreover, the exceptional subset of a $C_{0}$-operator is a topologically small subset of $\boldsymbol{K}$.

Let $H_{K}^{2}$ be the $H^{2}$ Hardy class of analytic functions in the unit disc $D=$ $\{z:|z|<1\}$ with values in the complex separable Hilbert space $K$. If $F(z)=$ $\sum_{n=0}^{+\infty} \psi_{n} z^{n} \quad\left(\psi_{n} \in K\right)$ is an element of $H_{K}^{2}$, then in the norm of $K$,

$$
\lim F(z)=F\left(e^{i x}\right), \quad z \rightarrow e^{i x} \text { nontangentially, }
$$

exists for almost every $e^{i x} \in \partial D=\{z:|z|=1\}$. If $G(z)=\sum_{n=0}^{+\infty} \xi_{n} z^{n}$ is also an element of $H_{K}^{2}$, then the inner product of $F$ and $G$ can be expressed as

$$
\langle F, G\rangle=\sum_{n=0}^{+\infty}\left(\psi_{n}, \xi_{n}\right)=\int_{\partial D}\left(F\left(e^{i x}\right), G\left(e^{i x}\right)\right) d m,
$$

where $($,$) denotes the inner product of K$ and $d m=(1 / 2 \pi) d x$ is the normalized Lebesgue measure on $\partial D([3],[4],[9])$.

The shift operator $S$ in $H_{K}^{2}$ is defined by

$$
S F(z)=z F(z)=\sum_{n=0}^{+\infty} \psi_{n} z^{n+1}
$$

and its adjoint $S^{\#}$ is given by

$$
S^{\#} F(z)=\frac{F(z)-F(0)}{z}=\sum_{n=0}^{+\infty} \psi_{n+1} z^{n} .
$$

Received by the editors November 13, 1970 and, in revised form, December 20, 1971. AMS 1970 subject classifications. Primary 47A15, 47A20, 47A60.

Key words and key phrases. Sz.-Nagy and Foias' functional calculus, Baire category

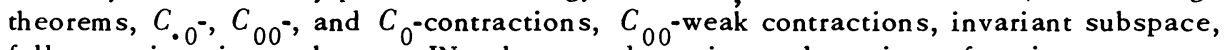
full-range invariant subspace, IN-subspace, determinant class, inner function-operator, IN-operator, minimal inner function, characteristic function, analytic direction, exceptional subset, quasi-similar transformation, Jordan model.

(1) This research was partially supported by National Science Foundation Grants GP-14255 and GU-3171. 
An invariant subspace is a subspace (i.e., a closed linear manifold) $M$ of $H_{K}^{2}$ such that $S M \subset M$. If $K=\mathbb{M}^{\perp}$, then $\mathcal{K}$ is invariant under $S^{\#}$ (we shall say that $\mathcal{K}$ is $S^{\#}$-invariant) and the operator $T: \mathcal{K} \rightarrow \mathcal{K}$ defined by

$$
T F=\mathbf{P}(S F),
$$

where $\mathbf{P}$ denotes the orthogonal projection of $H_{K}^{2}$ onto $K$, is a $C_{._{0}}$-contraction in the sense of [9]; i.e., $\|T\|=\|\mathbf{P S}\| \leqq 1$ and $T^{* n} \rightarrow 0$ strongly as $n$ tends to $\infty$. The first condition is clear and the second one follows from the fact that $T^{*}$ $=S^{\#} \mid \mathcal{K}\left(=S^{\#}\right.$ restricted to $\left.\mathcal{K}\right)$.

Moreover, we have

$$
T^{n} \mathbf{P}=\mathbf{P} S^{n}, \quad \text { for } n=0,1,2, \cdots
$$

Conversely, if $T$ is a $C_{.0}$-contraction of the complex separable Hilbert space $K$, then $T$ is unitarily equivalent to an operator of the type described above and (0.2) holds. This is a special case of the functional model of Sz.Nagy and Foias. In [9], these authors develop the following functional calculus:

In $u \in H^{\infty}$ (the Banach algebra of all bounded analytic functions in $D$ ) and $T=\mathrm{P} S$, then the map $u \rightarrow u(T)$ defined by

$$
u(T) F=\mathbf{P}(u F) \quad(F \in \mathcal{K})
$$

is an algebra homomorphism from $H^{\infty}$ into the space of all bounded linear operators in $\mathcal{K}$; moreover, $\|u(T)\| \leq\|u\|_{\infty}$.

In this functional model each property of the invariant subspace $M$ corresponds to a property of the operator $T$, and conversely. We are going to use these correspondences in order to obtain results about a particular class of contractions.

Using results in [9, Chapter III], the class $C_{0}$, originally defined by means of a quite complicated functional calculus, can be regarded as the subset of all those $C_{0}$-contractions such that $u(T)=0$ for some nonidentically zero function $u \in H^{\infty}$. It is known [9, Proposition III. 4.4] that if $T$ is a $C_{0}$-operator, then there exists an inner function $m_{T}$ (the minimal function of $T$ ) such that $u(T)=$ 0 if and only if $u \in m_{T} H^{\infty}$.

The existence of a nonexceptional vector was proved in [10] for the case when $K$ is a finite dimensional vector space, and this result plays a very important role in the theory of Jordan models [11].

Professor B. Sz.-Nagy notified us that he and C. Foias, have recently found a proof of the existence of a nonexceptional veçtor in the general case, and they have used this fact to extend the theory of Jordan models [13]. These results are partially analyzed in $\$ 3$, in connection with the study of the so-called weak contractions. 
The results of this article are partially contained in the author's thesis [4].

I. Preliminary results.

1. In [4] we have analyzed the properties of three families of invariant subspaces, namely, those included in the following definitions.

Definition $A$. An invariant subspace $\mathbb{M}$ is called a full-range invariant subspace if given any weakly measurable function $G\left(e^{i x}\right)$ defined on $\partial D$ with values in $K$, the condition $G\left(e^{i x}\right) \perp F\left(e^{i x}\right)$ in the sense of $K$ (a.e.), for all $F \in \mathbb{M}$, implies $G\left(e^{i x}\right)=0$ almost everywhere.

It follows from the Beurling-Lax-Halmos theorem [3, Lectures VI and VII] that $M$ is a full-range invariant subspace if and only if it can be expressed as

$$
M=\left\{U\left(e^{i x}\right) F\left(e^{i x}\right)=U F\left(e^{i x}\right): F \in H_{K}^{2}\right\},
$$

where $U$ is an inner function-operator; i.e., $U\left(e^{i x}\right)$ is a (weakly) measurable function defined on $\partial D$ with values in the set of all unitary operators in $K$ such that $U\left(e^{i x}\right) \phi \in H_{K}^{2}$, for all $\phi \in K$. Then $U$ is uniquely determined by $M$ up to right multiplication by a constant unitary operator. (Two inner function-operators are identified whenever they agree a.e. on $\partial D$ [3].)

An inner function-operator $U\left(e^{i x}\right)$ can be continued to an analytic operator valued function defined on $D$ such that $\|U(z)\|_{K} \leq 1$, for all $z \in D$, and $U\left(e^{i x}\right)=$ (strong) $\lim U(z)$, as $z \rightarrow e^{i x}$ nontangentially (see [4], [9]).

Definition $B$. An invariant subspace $\mathbb{N}$ is called an IN-subspace if there exists a (scalar) inner function $q$ such that $q H_{K}^{2} \subset \mathbb{M}$ [2], [4].

Definition $C$. An invariant subspace $\mathbb{M}=U H_{K}^{2}$ is called a subspace of the determinant class if it is a full-range invariant subspace and if $I-U^{*}(z) U(z)$ belongs to the (classical) trace class of operators in $K$, for all $z \in D$ [1], [4].

Denote by $\mathcal{F}^{\sim},(\mathrm{IN})^{\sim}$, and $(\mathrm{det})^{\sim}$ the classes of all invariant subspaces corresponding to the Definitions A, B, C, resp. Then we have: (det) $\sim($ IN) $\subset \mathcal{F}^{\sim}$ ((det), (IN) and $\mathcal{F}$ will denote the corresponding families of inner functionoperators). If $\operatorname{dim} K<\infty$, then (det) $=(\mathrm{IN})=\mathcal{F}$; on the other hand, if $K$ is infinite dimensional, then $($ det $) \neq($ IN $) \neq \mathcal{F}[4]$.

If $\mathbb{N}=U H_{K}^{2} \in(\mathrm{det})^{\sim}$, then there exists a constant unitary operator $X$ such that $U(z) X$ belongs to the classical determinant class of operators in $K$; in what follows, we are always going to assume that, if $\mathbb{M}=U H_{K}^{2}$ belongs to the determinant class, then the inner function-operator $U$ is chosen so that [determinant of $U(z)$ ] is well defined for all $z \in D$ (see [1], [4]); moreover, it will be assumed that det $U(z)$ (which is an inner function) is normalized in the sense that its first nonzero Taylor coefficient is positive. We shall make the same assumption for any inner function.

Let $\mathbb{M}=U H_{K}^{2} \in \mathcal{F}^{2}$; if $K_{1}=\mathbb{M} \cap K$, then we can write $K=K_{0} \oplus K_{1}$ (orthogonal direct sum) and 


$$
\mathbb{M}=H_{K_{1}}^{2} \oplus\left[\mathbb{M} \cap H_{K_{0}}^{2}\right]=H_{K_{1}}^{2} \oplus U_{0} H_{K_{0}}^{2}
$$

where $U_{0}$ is an inner function-operator with respect to the subspace $K_{0}$; moreover, $\left\|U_{0}(0) \phi\right\|_{K}<\|\phi\|_{K}$, for all $\phi \in K_{0}, \phi \neq 0$.

It follows from [9, Proposition VI, 3.5] that $\mathbb{M} \in \mathcal{F}^{\sim}$ if and only if $T \in C_{00}$; moreover, the inner function-operator $U_{0}(z)$ is equal (up to constant unitary factors. on both sides) to the characteristic function $\theta_{T}(z)$ of $T(=\mathbf{P S})$, i.e.

$$
U_{0}(z)=\theta_{T}(z)=\left.\left[-T+z D_{T}\left(I-z T^{*}\right)^{-1} D_{T}\right]\right|_{\mathscr{D}_{T}},
$$

where $D_{T}=\left(I-T^{*} T\right)^{1 / 2}, D_{T^{*}=}\left(I-T T^{*}\right)^{1 / 2}, \mathscr{D}_{T}=\overline{D_{T}(\mathcal{K})}, \mathscr{D}_{T^{*}}=\overline{D_{T^{*}}(\mathcal{K})}$ (the upper bar denotes topological closure; $\theta_{T}(z): \mathscr{D}_{T} \rightarrow \mathscr{D}_{T^{*}}$, for each $\left.z \in D\right)$.

Let $\mathbb{M}$ be an invariant subspace and let $F \in H_{K}^{2}$. Following M. J. Sherman [7], we shall say that $M$ contains the direction of $F$ if, for some scalar function $f \equiv 0, f F \in M$. Then, we have the following

Proposition 1.1 ([4, Chapter I]; see also [7]). (i) Let $\mathbb{M}=U H_{K}^{2} \in(\mathrm{IN})^{\sim}$; then there exists an inner function $q$ such that $r H_{K}^{2} \subset \mathbb{M}$ ( $r$ an inner function) if and only if $r \in q H^{\infty}$ (i.e., if and only if $q$ divides $r$; this fact will be denoted by $r<q$ ).

(ii) Similarly, if $\pi$ is an invariant subspace containing the direction of $F \in$ $H_{K}^{2}$, then there exists an inner function $p$ such that $p F \in \Re$ and $f \in H^{\infty}, f F \in \Re$ $\Rightarrow f \in p H^{\infty}$.

The function $q$ of (i) is precisely the minimal function $m_{T}$ of the introduction, and it will be called the minimal inner function (mif) of $\mathbb{N}$ (or $U$, or $T$ ). The function $p$ of (ii) is the minimal inner function of $F$ with respect to $\pi$ ( $p$ is the $\operatorname{mif}(\mathcal{T})$ of $F)$.

A relation between $q, U$ and $T$ is given by the following:

Theorem 1.2 ([4, Chapter V], [9, Chapter VI]). Let $U \in(\mathrm{IN})$ and let $q$ be its mif. The domain of analyticity of $U(z)\left(U^{-1}(z)\right.$, resp.) is the same as the domain of analyticity of $q(z)\left(q^{-1}(z)\right.$, resp. $)$; moreover, $U$ and $q\left(U^{-1}\right.$ and $q^{-1}$, resp. $)$ bave the same kind of isolated singularities.

In particular, the set $D \cap \sigma(T)$ ( $T$ defined as usual, $\sigma(T)$ denotes the spectrum of $T$ ) is precisely the set of all zeroes of $q(z)$.

Now we can state the following correspondences:

Proposition 1.3. The invariant subspace $M$ belongs to the class:

(i) $\mathcal{F}^{\sim}$ if and only if $T \in C_{00}$,

(ii) (IN) ${ }^{\sim}$ if and only if $T \in C_{0}$,

(iii) (det) ${ }^{-}$if and only if $T \in C_{00}$ and it is a weak contraction (i.e., trace $\left(I-T^{*} T\right)<\infty$ and $\left.D \not \subset \sigma(T)\right)$. 
Proof. The statements (i) and (ii) are clear from the previous results. Observe that $\left(I-T^{*} T\right): \mathcal{K} \rightarrow \mathcal{K}=\mathscr{D}_{T} \oplus \mathscr{D}_{T}^{1}$ is a nonnegative hermitian operator with kernel equal to $\mathfrak{D}_{T}^{\perp}$; thus we have

$$
\begin{aligned}
\operatorname{tr}\left(I_{K}-T^{*} T\right) & =\operatorname{tr}\left(\left[I_{K}-T^{*} T\right] \mid \mathfrak{T}_{T}\right)=\operatorname{tr}\left(I_{\mathscr{D}_{T}}-\theta_{T}^{*}(0) \theta_{T}(0)\right) \\
& =\operatorname{tr}\left(I_{K_{0}}-U_{0}^{*}(0) U_{0}(0)\right)=\operatorname{tr}\left(I_{K}-U^{*}(0) U(0)\right) .
\end{aligned}
$$

On the other hand, the characteristic function $T_{\alpha}=(T-\alpha)(I-\bar{\alpha} T)^{-1}$ is equal to $\theta_{T_{\alpha}}(z)=\theta_{T}((z+\alpha) /(1+\bar{\alpha} z))$, for any $a \in D$ (cf. [9, Chapters VI and VIII]) and $T_{a}$ is a weak contraction if and only if $T$ is so.

Using these facts, the following statements are clearly equivalent:

(1) $T$ is a weak $C_{00}$-contraction;

(2) $U \in \mathcal{F}$ and $\operatorname{tr}\left(I-U^{*}(0) U(0)\right)<\infty$;

(3) $U \in \mathcal{F}$ and $\operatorname{tr}\left(I-U^{*}(\alpha) U(\alpha)\right)<\infty$, for all $\alpha \in D$;

(4) $\mathbb{M} \in(\mathrm{det})^{-}$.

In fact, $(1) \Rightarrow(2) \Rightarrow(3) \Rightarrow(4) \Rightarrow(3)$ follow immediately from the definitions and previous arguments. It is also clear that (3) implies that $T \in C_{00}$ and $\operatorname{tr}\left(I-T^{*} T\right)<\infty$. Since $U \in(\mathrm{det}) \subset(\mathrm{IN})$, it follows from Theorem 1.2 that $D \not \subset$ $\sigma(T)$. Hence, $T$ is a weak $C_{00}$-contraction. Q.E.D.

Remark. The implication: $T$ is a weak $C_{00}$-contraction $\Rightarrow \mathbb{M} \in(\mathrm{det})^{\sim}$, is contained in [9]. The proof of [9, Theorem VIII. 1.1] is precisely the construction of the determinant of $\theta_{T}(z)$ !.

2. The next result is immediate ([3], [9]):

Lemma 1.4. Let $\mathbb{M}$ be an invariant subspace and let $T$ be defined by (0.1). Then, to each $T$-invariant subspace $\mathcal{K}_{0} \subset \mathcal{K}$ corresponds exactly one invariant

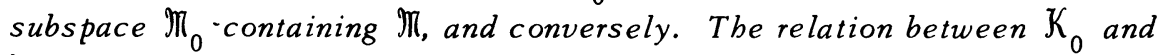
$\Pi_{0}$ is given by

(1) $K_{0}=K \cap \mathbb{M}_{0}$;

(2) $\mathbb{M}_{0}=\mathbb{M} \oplus \mathcal{K}_{0}=M \times K_{0}$ (Cartesian product).

Proposition 1.5 ([4, Chapter II], [9, Chapter VII $])$. Let $\mathbb{N}=U H_{K}^{2} \in(\mathrm{IN})^{\sim}$ with mif $q$. For any function $p$ such that $q<p$, define

$$
M_{p}=\left\{F \in H_{K}^{2}:(q / p) F \in M\right\}=U_{p} H_{K}^{2} .
$$

Then

(i) $\mathbb{M}_{\subset} \subset \mathbb{M}_{p} \in(\mathrm{IN})^{\sim}$ and the mif of $\mathbb{M}_{p}$ is equal to $p$.

(ii) If $q=\Pi_{n} q_{n}$ is any factorization of $q$ into pairwise coprime inner factors, then $\mathbb{M}^{n}=\bigcap_{n}^{n} \Re_{q_{n}}$ and $\mathcal{K}=\mathbb{M}^{\perp}=\bigvee_{n} K_{q_{n}}$, where $K_{q_{n}}=M_{q_{n}}^{\perp}$, $K_{q_{n}} \cap$ $K_{q_{m}}=\{0\}$ if $n \neq m\left(\bigvee_{n} \mathcal{K}_{q_{n}}\right.$ denotes the closed subspace spanned by the $\mathcal{K}_{q_{n}}$ 's). 
The most interesting case corresponds to the canonical factorization (see [4], [5]):

$$
q=b d s=\left(\prod_{k} b_{k}^{m} k\right)\left(\prod_{i} d_{j}\right) s
$$

where

(1) $b$ is the Blascbke product of $q, b_{k}$ is the elementary Blaschke factor corresponding to the zero $\lambda_{k} \in D\left(\lambda_{k} \neq \lambda_{b}\right.$ if $\left.k \neq b\right)$ and $m_{k}$ is the order of this zero,

(2) $d_{j}(z)=\exp \left\{l_{j}\left(z+e^{i \theta_{j}}\right) /\left(z-e^{i \theta_{j}}\right)\right\}, \quad 0 \leq \theta_{j}<2 \pi, \theta_{j} \neq \theta_{b}$ if $j \neq b, l_{j}>0$, $\Sigma_{j} l_{j}<\infty$, and $d=\Pi_{j} d_{j}$ is the discrete singular factor of $q$; finally,

(3) $s(z)=\exp \left\{\int_{\partial D}\left(z+e^{i y}\right) /\left(z-e^{i y}\right) d \nu(y)\right\}=e(z, \nu)$, where $\nu(y)$ is a measure given by a continuous nondecreasing function, which is singular with respect to Lebesgue measure in $[0,2 \pi] . s(z)$ is called the continuous singular factor of $q$.

As in [4], [7], the least common multiple (greatest common divisor, resp.) of two inner functions, $p$ and $q$, will be denoted by $p \wedge q(p \vee q$, resp.).

Lemma 1.6 ([4, Chapter II]). Let $\mathbb{M} \in \mathcal{F}^{-}, G \in H_{K}^{2}$ and assume that $\mathbb{M}$ contains the direction of $G$ with $\operatorname{mif}(\mathcal{M})$ of $G$ equal to $q=p r$, where $p$ and $r$ are inner functions. Then

$$
\mathbb{M}=U H_{k}^{2} \subset \prod_{p}=\left\{F \in H_{K}^{2}: r F \in \mathbb{M}\right\} \subset H_{k}^{2}
$$

and the mif $\left(M_{p}\right)$ of $G$ is equal to $r$.

Moreover, if $\prod_{p}=U_{p} H_{K}^{2}$ and $\mathbb{M}=U_{p} V_{p} H_{K}^{2}, U_{p}, V_{p} \in \mathcal{F}$ (to every invariant subspace containing $\mathbb{M}$ there corresponds a factorization of $U$ of this type; see [3], [8]), then $V_{p} \in(\mathrm{IN})$ and its mif is equal to $p$. Thus, the lattice of invariant subspaces

$$
\left\{\pi: \Re \subset \Re \subset H_{K}^{2}\right\}
$$

contains a sublattice isomorphic to the lattice of inner functions

$$
\{p: q<p<1\} \text {. }
$$

Proposition 1.7. Let $\mathbb{M}=U H_{K}^{2} \in(\mathrm{det})^{\sim}$ be an invariant subspace whose mif $q$ coincides with the determinant of $U$. Then the lattices (1.5) and (1.6) of Lemma 1.6 are identical.

Proof. The proof can be obtained by a modification of the argument given in [10, Theorem 1], or by the following argument based on results in [4, Chapter II]: Let $\pi=V H_{K}^{2}$ be any invariant subspace containing $\pi$ and let $U=V W$ be the corresponding factorization of the inner function-operat or $U$. Then $V, W \in($ det); moreover, if $p, p^{\prime}\left(r, r^{\prime}\right.$, resp.) are the mif and the determinant of $V$ (W, resp.), 
then $p^{\prime}=p s\left(r^{\prime}=r t\right.$, resp.; $s$ and $t$ are scalar inner functions $)$ and we have

$$
q=\operatorname{det} U=(\operatorname{det} V)(\operatorname{det} W)=p^{\prime} r^{\prime}=(p r)(s t) .
$$

On the other hand, $p r H_{K}^{2} \subset V\left(r H_{K}^{2}\right) \subset V W H_{K}^{2}=U ! I_{K}^{2}$. Therefore, $p r<q<(p r)(s t)$. We conclude that $s t=1$, and $p=$ mif of $V=\operatorname{det} V, r=\operatorname{mif}$ of $W=\operatorname{det} W$.

By Proposition 1.5, the mif of $\mathbb{M}_{p}=\left\{F \in H_{K}^{2}: r F \in \mathbb{M}\right\}=U_{p} H_{K}^{2}$ is equal to $p$; since the mif of $\pi$ is also equal to $p$, it is not hard to verify that

$$
\pi \subset M_{p}=\pi \vee p H_{K}^{2}=\bigcap\left\{\Re^{\prime}: M \subset \Re^{\prime}, p H_{K}^{2} \subset \Re^{\prime}\right\}
$$

The first part of the proof and the above inclusion imply that $p=\operatorname{det} U_{p}<$ $\operatorname{det} V=p$.

Hence $\operatorname{det} U=\operatorname{det} V$ and $\pi=M_{p}$. Q.E.D.

II. Exceptional subsets.

1. Definition D. Let $\pi \in(\mathrm{IN})^{-}$with mif $q$. The set

$$
\mathcal{E}(\mathbb{M})=\left\{F \in H_{K}^{2}: q_{F} \neq q, q_{F} \text { is the mif }(\mathbb{N}) \text { of } F\right\}
$$

will be called the exceptional subset of $H_{K}^{2}$ with respect to $\mathbb{M}$.

Theorem 2.1. Let $\mathbb{M} \in(\mathrm{IN})$ with mif $q=b d s=\Pi_{n} q_{n}$ (where the $q_{n}$ 's are nonconstant pairwise coprime inner functions). Then

(i) $\mathcal{E}(\mathbb{M})=\bigcup_{n} \mathcal{E}\left(\mathbb{M}_{q_{n}}\right)\left(\mathbb{M}_{q_{n}}\right.$ defined by (1.3));

(ii) $\mathcal{E}(\mathbb{M})=K_{\sigma} \times \mathbb{M}$ (Cartesian product), where $K_{\sigma}=\mathcal{E}(\mathbb{M}) \cap K=\mathbf{P}[\mathcal{E}(\mathbb{M})]$;

(iii) if $s=1$, then $\mathcal{E}(\mathbb{N})$ is a countable un ion of proper invariant subspaces and $K_{\sigma}$ is a countable union of proper $T$-invariant subspaces of $K$;

(iv) $\mathcal{E}(\mathbb{M})$ is a first category $F_{\sigma}$ subset of $H_{K}^{2}$ and $K_{\sigma}$ is a first category $F_{\sigma}$ subset of $\mathcal{K}$.

Note. We shall see later that the statement (iii) is sharp.

Proof. (i) Let $F \in \mathcal{E}(\mathbb{M})$ and let $p$ be its mif $(\mathbb{M})$; then $q<p$ and $q \neq p$. Therefore, there exists a factor $q_{n}$ of $q$ such that $q_{n}$ does not divide $p$; let $p=p_{n}{ }^{r}$, where $p_{n}=p \vee q_{n}$. Then $q_{n}<p_{n}, q_{n} \neq p_{n}$ and $q_{n} \wedge r_{n}=1$.

Therefore, the $w^{*}$-closed invariant subspace of $H^{\infty}, \Re=\left\{f \in H^{\infty}: f\left(p_{n} F\right) \in\right.$ $M_{q_{n}}$, contains $q_{n}$ and $r_{n}$; hence (see [3, Lecutre IV]) it also contains $r_{n} \vee$ $q_{n}=1$. Thus, $F \in E\left(M_{q_{n}}\right)$ and we conclude that

$$
E(\mathbb{M}) \subset \bigcup_{n} E\left(\mathbb{M}_{q_{n}}\right)
$$

Conversely, if $p_{n} F \in \mathbb{M}_{q_{n}}$, where $q_{n}<p_{n}$ for all $n$, and $p_{m} \neq q_{m}$, for some $m$, then

$$
\left(\prod_{n} p_{n}\right) F \in \mathbb{M} \Rightarrow \frac{q p_{m}}{q_{m}} F \in \mathbb{M} \Rightarrow F \in \varepsilon(\mathbb{M}) \Rightarrow \xi(\mathbb{M}) \supset \bigcup_{n} \varepsilon\left(\mathbb{M} q_{n}\right) .
$$


(ii) Let $F=F_{0}+F_{1} \in H_{K}^{2}$, where $F_{0}=\mathbf{P} F \in K$ and $F_{1}=(I-\mathbf{P}) F \in \mathbb{M}$, and let $p$ be an inner function.

Then, $p F \in \mathbb{M}$ if and only if $p F_{0} \in \mathbb{M}$ (since $\mathbb{M}$ is an invariant subspace, it is clear that $\left.p F_{1} \in \mathbb{M}\right)$.

Hence if $F \in \mathcal{E}(\mathbb{M})$, then $P F \in \mathcal{E}(\mathbb{M}) \cap K$, and therefore, $P[\mathcal{E}(\mathbb{M})] \subset \mathcal{E}(\mathbb{M}) \cap K$.

On the other hand, if $F \in \mathcal{E}(\mathbb{M}) \cap \mathcal{K}$ and $G \in \mathbb{M}$, then $F+G$ has the same mif (M) as $F$; hence $F+G \in \mathcal{E}(\mathbb{M})$ from which the result follows.

(iii) If $s=1$, then $q=\left(\Pi_{k} b_{k}^{m} k\right)\left(\Pi_{j} d_{j}\right)$ [as in (1.4)]. According to (i),

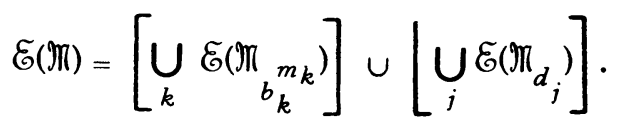

Observe that

$$
\mathcal{E}\left(\mathbb{M}_{b_{k}^{m_{k}}}\right)=\left\{F \in H_{k}^{2} ; b_{k}^{m_{k}-1} F \in \mathbb{b}_{k}^{m_{k}}\right\}=\mathbb{M}_{k}^{\prime \prime} .
$$

By Proposition 1.5 the mif of $\mathbb{M}_{b_{k}}^{m_{k}}$ is equal to $b_{k}^{m_{k}}$ and the mif of $\mathbb{M}_{k}^{\prime \prime}$ is equal to $b_{k}$; therefore, $m_{k}^{\prime \prime}$ is a proper invariant subspace of $H_{K}^{2}$.

Similarly, the mif of $\prod_{d_{i j}}$ is equal to $d_{j}\left(d_{j} \neq 1\right)$ and the mif of

$$
\prod_{j, n}^{\prime \prime}=\left\{F \in H_{K}^{2}: d_{j}^{(1-1 / n)} F \in \Re_{d_{j}}\right\}
$$

is equal to $d_{j}^{1 / n} \neq 1$; therefore $M_{j, n}^{\prime \prime}$ is a proper invariant subspace of $H_{K}^{2}$. Since $E\left(\mathbb{M}_{d_{j}}\right)$ is clearly equal to $\bigcup_{n=1}^{\infty} \mathbb{M}_{j, n}^{\prime \prime}$, we conclude that

$$
\mathcal{E}(\mathbb{M})=\left[\bigcup_{k} M_{k}^{\prime \prime}\right] \cup\left[\bigcup_{j} \bigcup_{n=1}^{\infty} \Re_{j, n}^{n}\right]
$$

has the desired property.

The statement relative to $K_{\sigma}$ follows from (ii) and the previous argument.

(iv) We are going to prove that $E(M)$ is always an $F_{\sigma}$ subset of $H_{K}^{2}$. According to (i),

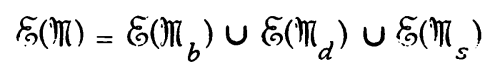

and we have already proved that $\mathcal{E}\left(m_{b}\right)$ and $\mathcal{E}\left(\Pi_{d}\right)$ are actually first category $F_{\sigma}$ subsets of $H_{K}^{2}$.

Let $s(z)=e(z, \mu)$; then $\mathcal{E}\left(\mathbb{M}_{s}\right)=\bigcup_{n=1}^{\infty} R_{s, n}$, where $\mathbb{R}_{s, n}=\left\{F \in H_{K}^{2}\right.$ : $e(z, \nu) F \in \mathbb{M}_{s}$, for some measure $\nu \leq \mu$, such that $\left.\|\nu\| \leq\|\mu\|-1 / n\right\}$.

Let $\mathcal{P}_{s, n}$ be the set of all those measures on $\partial D$ satisfying the above conditions. $\mathcal{L}_{s, n}^{s, n}$ is clearly a convex $w^{*}$-compact set of measures; hence if $F_{k}$ $\in R_{s, n}, k=1,2, \cdots, F_{k} \rightarrow F_{0}$ in $H_{K}^{2}$ and $\nu_{k} \in \mathcal{P}_{s, n}$ is the measure as sociated to $F_{k}$, then we can assume (passing if necessary to a subsequence) that $\nu_{k} \rightarrow$

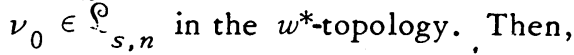

$$
\begin{aligned}
\left\|e\left(z, \nu_{k}\right) F_{k}-e\left(z, \nu_{0}\right) F_{0}\right\| & \leq\left\|\left[e\left(z, \nu_{k}\right)-e\left(z, \nu_{0}\right)\right] F_{0} !+\right\| e\left(z, \nu_{k}\right)\left[F_{k}-F_{0} ! \|\right. \\
& =\left\|\left[e\left(z, \nu_{k}\right)-e\left(z, \nu_{0}\right)\right] F_{0}\right\|+\left\|F_{k}-F_{0}\right\| .
\end{aligned}
$$


By hypothesis, $\left\|F_{k}-F_{0}\right\| \rightarrow 0$, as $k \rightarrow \infty$. On the other hand, $e\left(z, \nu_{k}\right) \rightarrow$ $e\left(z, \nu_{0}\right)$ uniformly on compact subsets of $D$, and therefore, $e\left(z, \nu_{k}\right) \rightarrow e\left(z, \nu_{0}\right)$ weakly in $H^{2}$ (actually, the sequence converges in $H^{2}$-norm). Since $e\left(z, \nu_{k}\right)$, $e\left(z, \nu_{0}\right)$ are inner functions, we conclude (cf. [4, Chapter II]) that

$$
\left\|e\left(z, \nu_{k}\right) F_{k}-e\left(z, \nu_{0}\right) F_{0}\right\| \rightarrow 0, \text { as } k \rightarrow \infty .
$$

Thus $e\left(z, v_{0}\right) F_{0} \in \mathbb{M}, F_{0} \in R_{s, n}$, and we conclude that $R_{s, n}$ is a closed subset of $H_{K}^{2}$.

Hence $\mathcal{E}\left(\pi_{s}\right)$ (and, therefore, $\mathcal{E}(\mathbb{N})$ ) is a $F_{\sigma}$ subset of $H_{K}^{2}$.

In order to prove that $\mathcal{E}\left(\Re_{s}\right)$ is a first category subset we only need to show that $H_{K}^{2} \backslash \mathcal{E}_{(}\left(\pi_{s}\right)$ is dense in $H_{K}^{2}$.

We shall need the following

Lemma 2.2 (M. J. Sherman, [8]). Let $\mathbb{N}$ be an invariant subspace containing the directions of $F_{1}, F_{2} \in H_{K}^{2}$ and let $q_{1}, q_{2}$ be the mif $(\pi)$ of $F_{1}, F_{2}$, resp. Then, the mif $(\mathbb{N})$ of $F=F_{1}+\lambda F_{2}$ is equal to $q=q_{1} \wedge q_{2}$ for all $\lambda \in \mathbf{C}$, except at most for a denumerable subset.

Corollary 2.3. (i) If $\mathfrak{\complement}=\bigvee\left\{F_{1}, F_{2}, \ldots, F_{N}\right\}$, and $\mathbb{M}$ contains the direction of $F_{j} \in H_{K}^{2}$ with mif (M) equal to $p_{j}, j=1,2, \ldots, N$, then there exists $F$ $\in \mathfrak{L}$ whose mif $(\mathbb{N})$ is equal to $p=p_{1} \wedge p_{2} \wedge \cdots \wedge p_{N}$.

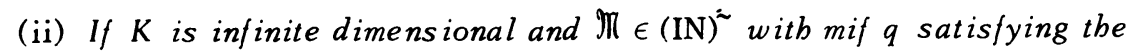
condition $q(0)>0$, then for every $\epsilon>0$ there exists a function $F_{\epsilon} \in H_{K}^{2}$ whose $\operatorname{mif}(\pi), q_{\epsilon}$, satisfies: $q_{\epsilon}(0)<q(0)+\epsilon$.

Furthermore, the set $G_{\epsilon}=\left\{F \in H_{K}^{2}: q_{F}(0)<q(0)+\epsilon\right\}$ (where $q_{F}$ denotes the mif $(\pi)$ of $F$ ) is dense in $H_{K}^{2}$.

Proof. (i) This follows from Lemma 2.2 by induction on $N$.

(ii) Let $\left\{\phi_{n}\right\}_{n=1}^{\infty}$ be an ONB of $K$, and let $K_{N}=\bigvee\left\{\phi_{1}, \ldots, \phi_{N}\right\}$. Then the mif of $M_{N}=\prod^{n} \cap H_{K_{N}}^{2}$ (considered as an invariant subspace of $H_{K_{N}}^{2}$ ) is equal to $p_{(N)}=p_{1} \wedge p_{2} \wedge \cdots \wedge p_{N}$.

By induction on $N$ we can obtain vectors $\psi_{N} \in K_{N}$ such that $p_{(N)}$ is the $\operatorname{mif}\left(M_{N}\right)=\operatorname{mif}(M)$ of $\psi_{N}, N=1,2, \ldots$.

It is clear that $\left|p_{(N)}(z)\right|$ is nonincreasing for each $z \in D$ and that $p_{(N)}$ converges to $q$ in $L^{2}$-norm and uniformly on compact subsets of $D$ [4, Chapter I]. Therefore, given any $\epsilon>0$ there exists $N$ such that $p_{N}(0)<q(0)+\epsilon$; then $\psi_{N}$ (chosen as above) satisfies our requirements.

From Lemma 2.2, the mif $(N)$ of $F_{\lambda}=\psi_{N}+\lambda F$ (for any fixed $F \in H_{K}^{2}$ ) is equal to $q_{F_{\lambda}}=p_{(N)} \wedge q_{F}$, for all but a countable subset of $\lambda$ 's. Hence

$$
q_{F_{\lambda}}(0) \leq p_{(N)}(0)<q(0)+\epsilon .
$$


This proves (ii). Q.E.D.

We are going to complete the proof of Theorem 2.1. To this end observe that Corollary 2.3, (ii) implies that $H_{K}^{2} \backslash R_{s, n}$ is dense in $H_{K}^{2}$. This proves that $\mathcal{G}\left(\pi_{s}\right)$ is a first category $F_{\sigma}$ subset of $H_{K}^{2}$.

As in (iii), the statement relative to $\mathcal{K}_{\sigma}$ follows from (ii) and the previous argument.

The proof is complete now.

Remarks. (a) The statement of Lemma 2.2 cannot be improved. In fact, if the inner function $q$ admits the expression $q=\Pi_{n=1}^{\infty} q_{n}$ (where the $q_{n}$ 's are nonconstant pairwise coprime inner functions), then we can construct an invariant subspace $\mathbb{M} \in(\mathrm{IN})^{\sim}$ with mif $q$, so that the set of exceptional functions $\omega_{k}=$ $\phi_{1}+\lambda_{k} \phi_{2}$ (where $\phi_{1}, \phi_{2}$ denote the constant functions $F_{j}\left(e^{i x}\right) \equiv \phi_{j}, \phi_{j} \in K$, $j=1,2)$ of the lemma is in one-to-one correspondence with an arbitrary given denumerable family of complex numbers $\left\{\lambda_{k}\right\}_{k=1}^{\infty}$. More generally, if $\operatorname{dim} K>1$ and $\left\{K_{j}\right\}_{j=1}^{\infty}$ is any family of proper closed subspaces of $K$, then the mif of $\mathbb{M}=$ $\bigcap_{j=1}^{\infty} \mathbb{M}_{j}^{j}$, where

$$
\Re_{j}=\left[P_{j}+\left(I-P_{j}\right) q_{j}\right] H_{K}^{2}
$$

( $P_{j}$ denotes the orthogonal projection of $K$ onto $K_{j}$ ), are equal to $q, q_{j}$, resp, and the exceptional subset $\mathcal{E}(\mathbb{N})$ satisfies the condition

$$
\left.\mathcal{E}(\mathbb{N}) \cap K=\left[\bigcup_{j=1}^{\infty} \mathcal{E}_{(\pi)}\right)\right] \cap K=\bigcup_{j=1}^{\infty} K_{j} .
$$

(b) If $\mathbb{M}_{1} \mathbb{M}_{1} \cap \mathbb{M}_{2} \in(\mathrm{IN})^{\sim}$, the mif of $\mathbb{M}_{j}$ is $q_{j}, j=1,2$, but $q_{1} \vee q_{2} \neq 1$, then $\mathscr{E}(\mathbb{M}) \subset \mathcal{E}\left(\mathbb{M}_{1}\right) \cup \mathcal{E}\left(\mathbb{M}_{2}\right)$ and the inclusion can be strict, in general. For example, if $K=\mathbf{C}^{2}$,

$$
\Re_{1}=\left[\begin{array}{cc}
e^{i x} & 0 \\
0 & 1
\end{array}\right] H_{K}^{2}, \quad \Re_{2}=\left[\begin{array}{ll}
1 & 0 \\
0 & e^{i x}
\end{array}\right] H_{K}^{2},
$$

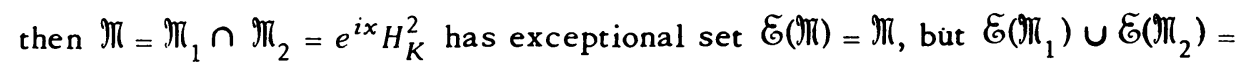
$\pi_{2} \cup \pi_{1} \neq \pi$

2. We are going to complete our results about exceptional subsets.

Proposition 2.4. Let $\mathbb{M} \in(\mathrm{IN})^{\sim}$ with mif $q=$ b.r. Then

(i) if $F \in \mathcal{E}(\mathbb{M})$, then closure $\left\{f F: f \in H^{\infty}\right\} \subset \mathcal{E}(\mathbb{M})$;

(ii) $\mathcal{E}(\mathbb{N})$ is always a (not necessarily denumerable) union of invariant subspaces; K);

(iii) if $r$ is nonconstant, then $\mathcal{E}(\mathbb{M})$ is dense in $H_{K}^{2}$ (and $\mathcal{K}_{\sigma}$ is dense in (iv) $H_{K}^{2}=\bigvee\left[H_{K}^{2} \backslash \mathcal{E}(\Re)\right], K=\bigvee\left[K \backslash K_{\sigma}\right]$ 
Moreover, if $q$ is not a power of an elementary Blaschke factor, then

$$
H_{K}^{2}=\bigvee \varepsilon(\pi), \quad K=\bigvee K_{\sigma},
$$

(v) $\mathcal{K}_{\sigma}=\{0\}$ if and only if there exist $\lambda \in D$ and a subspace $K_{0} \subset K, K_{0} \neq$ $\{0\}$ such that

$$
\mathcal{K}=\left\{(1-\bar{\lambda} z)^{-1} \phi: \phi \in K_{0}\right\} .
$$

In this case $\mathbb{M}=\left[\left(I-P_{0}\right)+(\lambda-z) /\left(1-\bar{\lambda}_{z}\right) P_{0}\right] H_{K}^{2}$, where $P_{0}$ is the orthogonal projection of $\mathcal{K}$ onto $\mathcal{K}_{0}$.

Proof. (i) Since $M$ is invariant

$$
q_{F} F \in \Re \Rightarrow f\left(q_{F} F\right)=q_{F}(f F) \in \pi, \text { for all } f \in H^{\infty} .
$$

Now the result follows from the fact that $M$ is closed.

(ii) This follows immediately from (i). In a more explicit form, we have

$$
\varepsilon(\mathbb{T})=\bigcup\left\{M_{p}: q \bar{p} \text { is a proper divisor of } q\right\} \text {. }
$$

(iii) Let $r=e(z, \mu)$; then

$$
\left.E(N) \supset E_{(N)}\right) \supset \bigcup_{n=2}^{\infty}\left[e(z, \mu / n) H_{K}^{2}\right],
$$

and this union of invariant subspaces is dense in $H_{K}^{2}$.

(iv) The first part is an immediate consequence of Theorem 2.1. Assume that $q(z)$ is not of the form $b^{N}(z, \lambda)$, for some $\lambda \in D$ and some nonnegative integer $N$, then we have two different cases:

(1) $\sigma(T)$ contains more than one point. Then $q$ can be factored as $q=p \cdot r$, where $p$ and $r$ are nonconstant coprime inner functions.

Let $\mathbb{M}_{p}, \mathbb{M}_{r}$ be defined by (1.3). Then $\mathbb{M}_{p} \cup \mathbb{M}_{r} \subset \mathcal{E}\left(\Re_{)}\right)$and

$$
M_{D} \vee M_{r}=\left[m_{D}^{\perp} \cap M_{r}^{\perp}\right]^{1}=H_{K}^{2}
$$

(2) $\sigma(T)$ consists of one isolated point of $\partial D$; in this case $q(z)=d(z, l, \theta)$ for some $l>0$ and $e^{i \theta} \in \partial D$. According to (iii) $\mathcal{E}(\mathcal{N})$ is dense in $H_{K}^{2}$ and, by Theorem 2.1, (ii) $K_{\sigma}$ is dense in $K$. This proves (2.1).

(v) Assume that $K_{\sigma}=\{0\}$. Then the argument of (iv)(1) implies that $q$ cannot be factored in a nontrivial way; i.e., $q=1$ or $q(z)=b(z, \lambda)$, for some $\lambda \epsilon$ $D$. In the first case, $\mathcal{K}=\{0\}$ and $\mathcal{K}_{\sigma}=\varnothing$; the second case and the remaining statements are contained in [4, Ghapter III].

From Theorem 2.1 we get

Corollary 2.5. Let $\mathbb{M}=U H_{K}^{2} \in \mathcal{F}^{\sim}$ and let $q$ be an inner function. The following two statements are equivalent. 
(i) There exists $F \in H_{K}^{2}$ whose mif $(\mathbb{N})$ is $q$;

(ii) $U$ can be factored as $U=W \cdot B$, where $W \in \mathcal{F}$ and $B$ is an IN-operator with mif $q$.

Proof. (i) $\Rightarrow$ (ii). This follows from Lemma 1.6.

(ii) $\Rightarrow$ (i). Assume that $U=W \cdot B$ and take $F=W \cdot G$, where $G \in H_{K}^{2}$ is any function such that its $\operatorname{mif}(B)$ is equal to $q$. Then the mif $(\mathbb{N})$ of $F$ is also $q$.

3. Most of the previous results are still valid when $H_{K}^{2}$ is replaced by some suitable (closed) subspace; namely

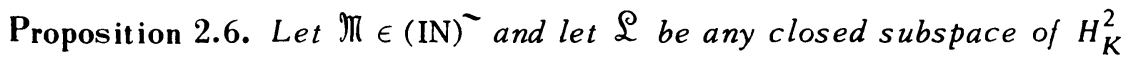
such that

$$
\mathcal{K}, \subset \bigvee_{n=0}^{\infty} S^{n}(\bigodot)
$$

Then $\mathcal{E}^{\prime \prime}(\mathbb{M})=\mathcal{E}(\mathbb{M}) \cap \mathfrak{Q}$ is a first category $F_{\sigma^{-s u b s e t}}$ of $\mathscr{\&}$.

Note. Observe that $\bigvee_{n=0}^{\infty} S^{n}(\mathcal{K})=H_{K_{0}}^{2}$ (defined by (1.1)).

Proof. It is clear from Theorem 2.1 that $\mathscr{E}^{\prime \prime}(\Re)$ is an $F_{\sigma^{-}}$subset of $\mathfrak{L}$.

Let $\left\{F_{k}\right\}_{k \in J}$ be on ONB of $\mathcal{Q}$ and let $p_{k}$ be the mif $(\mathbb{N})$ of $F_{k}$. If $p_{(N)}=$ $p_{1} \wedge p_{2} \wedge \ldots \wedge p_{N}$, then the limit

$$
p(z)=\lim p_{(N)}(z) \quad(N \rightarrow \infty)
$$

defines an inner divisor of the mif $q$ of $\pi$.

Since $K \subset \bigvee_{n=0}^{\infty}\left\{S^{n}\left(F_{k}\right): k \in J\right\}$ and, clearly, $p\left(S^{n} F_{k}\right) \in \mathbb{M}$ for all $k \in J$ and all $n=0,1,2, \ldots$, it follows that $p \mathcal{K} \subset \mathbb{M}$; therefore, $p H_{K}^{2} \subset \mathbb{M}$, i.e., $q$ divides $p$. Hence $q=p$.

On the other hand, a standard argument shows that the set $\mathscr{L}_{(N)}=\{F \in \mathfrak{L}$; $q_{F}$ divides $\left.p_{(N)}\right\}$ is dense in $\mathcal{L}$.

Using these two facts it is not hard to conclude (by a formal repetition of the proof of Theorem 2.1) that the complement of $\mathcal{E}^{\prime \prime}(\mathbb{N})$ in $\mathscr{Q}$ is a dense subset of $\mathcal{L}$.

In [7], M. J. Sherman conjectured that any invariant subspace containing all directions is necessarily an IN-subspace. This result was recently proved by Sz.-Nagy and Foiaș ([12]). Using Sz.-Nagy and Foiaș techniques, Sherman proved a stronger result: The conclusion remains valid if we merely assume that $\pi$ contains all the directions corresponding to a subspace $\mathscr{Q}$ such that $H_{K}^{2}=$ $\bigvee_{n=0}^{\infty} S^{n}(£)[8]$. A slightly stronger form of this theorem is given by the following:

Theorem 2.7. Let $\mathbb{M}$ be an invariant subspace containing all the directions of the functions $F \in \Omega$, where $\Omega$ is a second category subset of a subspace $\mathcal{L}$ satisfying (2.2). Then $\mathbb{M}$ is an IN-subspace. 
Proof. It is clear from the definition of $\mathcal{Q}$ and the properties of $\Omega$ that

$$
\mathcal{K}=\mathbb{M}^{\perp}=\bigvee\left\{\bigvee_{n=0}^{\infty} \mathbf{P} S^{n} F=\bigvee_{n=0}^{\infty} T^{n} \mathbf{P} F: F \in \Omega\right\} .
$$

Let $\mathcal{K}_{F}=\bigvee_{n=0}^{\infty} T^{n} \mathbf{P} F, M_{F}=K_{F}^{\perp}(F \in \Omega)$. By hypothesis, $\mathbb{M}_{F} \in(\mathrm{IN})^{\sim}$ und (2.3) implies that $\mathbb{M}=\bigcap\left\{\mathbb{M}_{F}: F \in \Omega\right\}$.

Let $q_{F}$ be the mif $(\Re)$ of $F \in \Omega$. If there exists an inner function $q$ such that $q<q_{F}$, for all $F \in \Omega$, then it is not hard to see that $q \Omega \subset \mathbb{M}$, and this implies

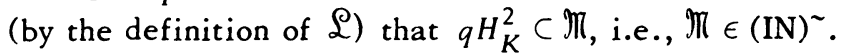

Assume that there is no such a function $q$; then we can choose a sequence $\left\{F_{n}\right\} \subset \Omega$ such that, if $p_{n}$ is the mif $(\mathbb{M})$ of $F_{n}$, then $p_{(N)}=p_{1} \wedge p_{2} \wedge \cdots \wedge p_{N}$ converges to zero uniformly on compact subsets of $D$. By our hypothesis on $\Omega$, it is not hard to deduce from Proposition 2.6 that the set

$$
\Omega^{\prime}=\Omega\left\lfloor\left[\bigcup_{n=1}^{\infty} E\left(M_{F_{n}}\right)\right]\right.
$$

cannot be empty!

Pick any function $F \in \Omega^{\prime}$. It follows from the definition of $\Omega^{\prime}$ that $p_{(N)}$ divides the mif $(M)$ of $F$ for all values of $N$, which is clearly impossible. Therefore, $M \in(I N)^{2}$.

If we omit the condition (2.2), then we have

Theorem 2.8. Let $M$ be an invariant subspace containing all the directions of the functions $F \in \Omega$, where $\Omega$ is a second category subset of a subspace $\mathcal{L}$. Then there exists an inner function $p$ satisfying the conditions:

(1) $p \mathfrak{L} \subset \mathbb{M}$.

(2) $q \mathfrak{L} \subset \mathbb{M}(q$ an inner function $) \Rightarrow p$ divides $q$.

(3) The set $\left\{F \in \mathscr{Q}: p\right.$ does not divide $q_{F}=$ the mif $(\mathbb{M})$ of $\left.F\right\}$ is a first category $F_{\sigma^{-s u b s e t}}$ of $\mathscr{\complement}$.

Proof. Let $\pi=\bigvee_{n=0}^{\infty} s^{n}(£) . ~ \pi$ is an invariant subspace and therefore, by the Beurling-Lax-Halmos theorem (see [3, Lecture V]) can be written as $\pi=$ $A H_{K_{1}}^{2}$, where $K_{1}$ is a suitable complex separable Hilbert space and $A$ is a function defined on $\partial D$ (a.e.) whose values are isometries of $K_{1}$ into $K$ and satisfying the condition: $A \psi \in H_{K_{1}}^{2}$, for all $\psi \in K_{1}$.

The operator $A$ commutes with the multiplication by $e^{i x}$ and it maps $H_{K_{1}}^{2}$ isometrically onto $\pi$. Hence there exist subspaces $\pi_{1}, \varrho_{1}$ contained in $H_{K_{1}}^{2}$ such that $A: \mathbb{K}_{1} \rightarrow \mathbb{M} \cap \Re_{;} A: \mathfrak{L}_{1} \rightarrow \mathfrak{\&}$ (isometrically onto).

Moreover, if $F=A F_{1}\left(F_{1} \in H_{K_{1}}^{2}\right)$, then $p F \in \mathbb{M}$ if and only if $p F_{1} \in \mathbb{M}_{1}$ and the subset $\Omega_{1}=\left\{F_{1} \in H_{K_{1}}^{2}: A F_{1} \in \Omega\right\}$ is a second category subset of $\mathscr{L}_{1}$.

Then, by Theorem 2.7, $M_{1}$ is an IN-subspace of $H_{K_{1}}^{2}$. Let $p$ be the mif of 
$M_{1}$; then $p$ clearly satisfies the conditions (1) and (2). Furthermore, $\{F \in \mathcal{L}: p$ does not divide $\left.q_{F}\right\}=A\left[\mathcal{G}\left(\Re_{1}\right)\right]$; hence $p$ also satisfies (3).

An example. Theorem 2.7 is almost sharp. Let $\mathbb{N}=U H_{K}^{2}$ be the full-range invariant subspace defined by the "diagonal" operator $U$, where $U \phi_{n}=z^{n} \phi_{n}$ $\left(\left\{\phi_{n}\right\}_{n=1}^{\infty}\right.$ is an ONB of $\left.K\right)$. Then $\mathbb{N}$ contains the analytic direction of all analytic polynomials whose coefficients are finite linear combinations of the $\phi_{n}$ 's (the set of all these polynomials is dense in $H_{K}^{2}$ ). However, $M$ is not an IN-subspace.

Now we are in a position to prove that the statement (iii) of Theorem 2.1 is sharp, i.e.

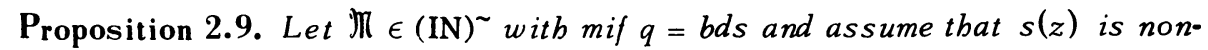
constant. Then $\mathcal{E}(\pi)$ cannot be written as a countable union of subspaces of $H_{K}^{2}$.

We shall need the following rather technical lemma:

Lemma 2.10. Let $\mu$ be a positive Borel measure on $\partial D$ containing no atoms and let $\left\{E_{n}\right\}_{n=1}^{\infty}$ be a sequence of $\mu$-measurable subsets such that $\mu\left(E_{n}\right)>0$. Then there exists a $\mu$-measurable subset $E$ such that $(1) \mu(E)>0 ;(2) \mu\left(E_{n} \backslash E\right)$ $>0$, for all $n=1,2, \cdots$.

Proof. Since $\mu$ contains no atoms, we can, given the sequence $\left\{E_{n}\right\}_{n=1}^{\infty}$, obtain a new sequence of $\mu$-measurable subsets $\left\{E_{n}^{\prime}\right\}_{n=1}^{\infty}$ such that

(a) $E_{n}^{\prime} \subset E_{n}$ for each $n$,

(b) $0<\mu\left(E_{n}^{\prime}\right)<\epsilon / 2^{n}$,

where $\epsilon$ is any positive number less than $\|\mu\|$.

Then the set $E=\partial D \backslash \bigcup_{n=1}^{\infty} E_{n}^{\prime}$ satisfies our requirements.

Proof of Proposition 2.9. Let $s(z)=e(z, \mu)$ and assume that

$$
\xi\left(\pi_{s}\right)=\bigcup_{n=1}^{\infty} \varrho_{n} \text {, }
$$

where $\mathcal{L}_{n}$ is a subspace of $H_{K}^{2}(n=1,2, \ldots)$.

It follows from Theorem 2.8 that, for each $n, \mathscr{L}_{n}$ is contained in an IN-subspace of the form $\mathbb{M}_{n}=\left\{F \in H_{K}^{2}: e\left(z, \mu_{n}\right) F \in \mathbb{M}\right\}$, where $\mu_{n}$ is a measure on $\partial D$ such that $d \mu_{n}=b_{n}(t) d \mu, 0 \leq b_{n}(t) \leq 1$ (a.e., $\left.d \mu\right)$ and

$$
\int_{\partial D} b_{n}(t) d \mu=\int_{D} d \mu_{n}=\left\|\mu_{n}\right\|<\|\mu\| \text {. }
$$

Since $\mathbb{M}_{n} \subset \mathcal{E}\left(\Re_{s}\right)$, we can assume that

$$
\mathcal{E}\left(\pi_{s}\right)=\bigcup_{n=1}^{\infty} \Re_{n}
$$

Let $E_{n}=\left\{t \in[0,2 \pi]: b_{n}(t) \neq 1\right\}$; then $\left\{E_{n}\right\}_{n=1}^{\infty}$ is a sequence of $\mu$-measurable subsets of $\partial D$ satisfying the conditions of Lemma 2.10. Let $E$ be a $\mu$ - 
measurable subset of $\partial D$, chosen as in the lemma and let $d \nu=\chi_{E}(t) d \mu$, where $\chi_{E}(t)$ is the characteristic function of $\partial D \backslash E$.

Claim. If $\Re_{=}\left\{F \in H_{K}^{2}: e(z, \nu) F \in \mathbb{M}_{s}\right\}$ then

$$
r \not \bigcup_{n=1}^{\infty} \pi_{n} \text {. }
$$

In fact, we have the following sequence of implications: $\pi \subset \bigcup_{n=1}^{\infty} \Re_{n} \Rightarrow$ $\pi \subset \Re_{n_{0}}$, for some $n_{0} \Rightarrow \nu<\mu_{n_{0}} \Rightarrow \chi_{E}(t) \leq b_{n_{0}}(t)$ (a.e., $\left.d \mu\right)$. However, by our choice of $E$, this last inequality is false for all values of $n$.

Therefore $\pi \not \subset \bigcup_{n=1}^{\infty} \pi_{n}$; since $\pi$ is clearly contained in $\mathscr{E}\left(\Re_{s}\right)$, we conclude that $\mathcal{E}\left(\pi_{s}\right) \neq \bigcup_{n=1}^{\infty} \mathfrak{Q}_{n}$, contradicting our assumption.

Since (by Theorem 2.1, (iii)) $\mathscr{E}(\mathbb{N})=\mathcal{E}\left(\mathbb{N}_{s}\right) \cup \mathcal{E}^{\prime}$, where $\mathcal{E}^{\prime}$ is a countable union of subspaces, we conclude that $\mathscr{E}(\mathcal{M})$ itself cannot be written as a countable union of subspaces.

4. We shall complete this section with two miscellaneous results.

Proposition 2.11. Let $M \in(\mathrm{IN})^{\sim}$ with mif $q$ and let $\mathcal{Q}$ be a closed subspace of $H_{K}^{2}$ satisfying the condition (2.2). Then there exists an orthonormal bas is $\left\{F_{n}\right\}$ of $\mathcal{Q}$ such that the mif $(\mathbb{M})$ of $F_{n}$ is equal to $q$, for all $n=1,2, \ldots$.

Proof. According to Proposition 2.6, we can find a function $G_{1} \in \mathscr{Q}^{1}=\{F \in$ $\mathcal{L}:\|F\|=1\}$ so that the mif $(\pi)$ of $G_{1}$ is $q$. Assume that $\left\{F_{1}, F_{2}, \ldots, F_{n-1}\right.$, $\left.G_{n}\right\}$ is an orthonormal system such that the mif $(\mathbb{N})$ of $F_{j}, j=1,2, \ldots, n-1$, and $G_{n}$ are equal to $q$. Let $G_{n+1}^{\prime} \in \mathfrak{L}^{1}, G_{n+1}^{\prime} \perp \bigvee\left\{F_{1}, F_{2}, \ldots, F_{n-1}, G_{n}\right\}$. Applying Lemma 2.2 to the functions $\left(\alpha G_{n}+\beta G_{n+1}^{\prime \prime}\right),\left(\beta G_{n}-\alpha G_{n+1}^{\prime}\right), \alpha, \beta \geq 0, \alpha^{2}+\beta^{2}=1$, we can get a pair

$$
F_{n}=\alpha_{n} G_{n}+\beta_{n} G_{n+1}^{\prime}, \quad G_{n+1}=\beta_{n} G_{n}-\alpha_{n} G_{n+1}^{\prime}
$$

such that the mif $(M)$ of $F_{n}, G_{n+1}$ are equal to $q$.

Let $\left\{E_{n}\right\}$ be any given ONB of $\mathscr{Q}$ and let $G_{1}$ be chosen as above. If $E_{1}=$ $\lambda G_{1}$ for some $\lambda \in \partial D$, take $G_{2}^{\prime}=E_{2}$; if $E_{1}$ and $G_{1}$ are linearly independent, then take

$$
G_{2}^{\prime}=\left(E_{1}-\left\langle E_{1}, G_{1}\right\rangle G_{1}\right)\left\|E_{1}-\left\langle E_{1}, G_{1}\right\rangle G_{1}\right\|^{-1}
$$

and so on. Now the result follows by induction on $n$.

The statement (i) of Proposition 2.4 clearly implies that if $F \in K_{\sigma}$ then it cannot be a cyclic vector for T. On the other hand, B. Sz.-Nagy and C. Foias, have proved [13] that if $F \in \mathcal{K} \backslash \mathcal{K}_{\sigma}$ is a cyclic vector for $T$, then every element of $\mathcal{K} \backslash K_{\sigma}$ is a cyclic vector; hence we have

Corollary 2.12. Let $T$ be a $C_{0}$-operator and assume that $T$ bas a cyclic vector. Then the set of all cyclic vectors of $T$ forms a $G_{\delta^{-}}$-dense subset of $K$. 
III. Weak $C_{00}$-contractions and Jordan models.

1. We shall need some definitions:

Let $T$ be a bounded linear operator in the (complex separable) Hilbert space $\mathcal{K}$ and let $\Xi$ be a subset of $\mathcal{K}$. The (finite or denumerable) cardinal number

$$
\mu_{T}=\inf \left\{\operatorname{cardinal}(\Xi): \mathcal{K}=\bigvee_{n=0}^{\infty} T^{n}(\Xi)\right\}
$$

is called the multiplicity of $T$ (see [11]). For example, $\mu_{T}=1$ means that $T$ has a cyclic vector.

If $T^{\prime}$ is a bounded linear operator in $\mathcal{K}^{\prime}$ (another Hilbert space) and there exists a quasi-invertible operator $X: \mathcal{K} \rightarrow \mathcal{K}^{\prime}$ (i.e., $\operatorname{ker}(X)=\{0\}$ and range $(X)$ is dense in $\mathcal{K}^{\prime}$ ) such that $T^{\prime} X=X T$, we shall write $T \prec T^{\prime}$ or $T^{\prime} \succ T$.

We have the following properties [11]:

(1) $T^{\prime}>T$ if and only if $T^{*}>T^{\prime *}$;

(2) $T^{\prime}>T$ and $T^{\prime \prime}>T^{\prime}$ imply $T^{\prime \prime}>T$;

(3) $T^{\prime}>T$ implies $\mu_{T} \geq \mu_{T^{\prime}}$;

(4) if $T^{\prime}$ and $T$ are $C_{00}$-contractions such that $T^{\prime}>T$, and $u \in H^{\infty}$, then $u\left(T^{\prime}\right)>u(T)$;

(5) if $T^{\prime}, T$ are $C_{0}$-contractions and $T^{\prime}>T$, then $m_{T}=m_{T^{\prime \prime}}$.

If $T^{\prime}>T$ and $T>T^{\prime}, \cdot^{\prime}$ - he operators $T$ and $T^{\prime}$ are said to be quas $i-$ similar.

Definition E. Let $\left\{q_{n}\right\}_{n=1}^{\omega}$ be a finite or infinite sequence of nonconstant inner functions satisfying the condition $q_{1}<q_{2}<\ldots<q_{n}<\ldots<1$. Then the Jordan operator corresponding to the sequence $\left\{q_{n}\right\}$ is the $C_{0}$-contraction associated to the invariant subspace

$$
\Pi=\bigoplus_{n}\left(q_{n} H^{2} \phi_{n}\right)
$$

where $\left\{\phi_{n}\right\}_{n=1}^{\omega}$ is an ONB of some Hilbert space $K^{\prime}$.

This operator will be denoted by

$$
J\left(q_{1}\right) \oplus J\left(q_{2}\right) \oplus \cdots \oplus J\left(q_{n}\right) \oplus \cdots, \quad \text { or } \quad J\left(q_{1}, q_{2}, q_{3}, \cdots q_{n}, \ldots\right) .
$$

The (finite or denumerable) cardinal number $\omega$ will be called the length of the operator $J$ (see [11]).

It is clear that the invariant subspace $\mathbb{N}$ is actually an IN-subspace with mif $q_{1}$.

It was proved in [11], [13] that if $T \in C_{0}$ then $\mu_{T}=\mu_{T} *$ and if $\mu_{T}=N<$ $\infty$, then $T$ is quasi-similar to a unique Jordan operator (the Jordan model of $T$ !) $J\left(q_{1}, \ldots, q_{N}\right)$ of length $N$.

We are going to extend the notion of Jordan model to a particular class of $C_{0}$-operators. Some of the proofs of the results of this section can be obtained 
from those in [10] and [11] (with perhaps a few formal modifications). In these cases, we shall merely quote the corresponding reference.

Let $(\mathrm{IN})_{B}=\{U \in(\mathrm{IN})$ : the mif of $U$ is a Blaschke product $\}$ and let (det) ${ }_{B}=$ $($ det $) \cap(\mathrm{IN})_{B}\left((\mathrm{IN})_{B}^{\sim}\right.$ and $(\mathrm{det})_{B}^{\tilde{\alpha}}$ are similarly defined $)$.

Lemma 3.1. Let $\mathbb{M}=U H_{K}^{2} \in(\mathrm{IN})_{B}^{\sim}$ with mif $q=b_{\lambda}^{N}$ (i.e., $q$ is the power of order $N \geq 1$ of an elementary Blaschke factor having its zero at the point $\lambda \in D$ ), and assume that $T$ bas a cyclic vector $G$. Then $\mathbb{M} \in(\mathrm{det})_{B}^{\sim}$ and $\operatorname{det} U=q$.

Proof. Observe that $\mathcal{K}=\mathbb{M}^{\perp} \subset\left[b_{\lambda}^{N} H_{K}^{2}\right]^{\perp}$; hence $G=\sum_{n=0}^{N-1} g_{\lambda} b_{\lambda}^{n} \psi_{n}$, where $g_{\lambda}(z)=\left(1-|\lambda|^{2}\right)^{1 / 2} /(1-\lambda z)$ and $\psi_{0}, \ldots, \psi_{N-1} \in K$.

Hence

$$
\mathcal{K}=\bigvee_{n=0}^{\infty} T^{n} G=\bigvee_{n=0}^{\infty}(T-\lambda)^{n} G=\bigvee_{n=0}^{\infty} b_{\lambda}^{n}(T) G=\bigvee_{n=0}^{N-1} b_{\lambda}^{n}(T) G
$$

is a finite dimensional space and therefore $\pi \in(\mathrm{det})_{B}^{-}$and $\operatorname{det} U$ is a Blaschke product of degree less than or equal to $N$ ([4, Chapter III]). Using this result and the relation $\operatorname{det} U<q$ we conclude that $\operatorname{det} U=q$ and $\operatorname{dim}(\mathcal{K})=N$.

Corollary 3.2. Let $\mathbb{M}=U H_{K}^{2} \in(\mathrm{IN})_{B}^{\sim}$ with mif $q=\Pi_{k} b_{k}^{m_{k}}$, and assume that $T$ admits a cyclic vector. Then $\Pi \in \in(\mathrm{det})_{B}^{\sim}$ and $\operatorname{det} U=q$.

Proof. By Theorem 1.2, $\sigma(T)=$ closure $\left\{\lambda_{k}: \lambda_{k}\right.$ is the zero of $\left.b_{k}(z)\right\}$. Fix $k$ and let $\Gamma_{k} \subset D \backslash \sigma(T)$ be a (positively oriented) circle separating $\left\{\lambda_{k}\right\}$ from $\sigma(T) \backslash\left\{\lambda_{k}\right\} ;$ then

$$
P_{k}(T)=\frac{1}{2 \pi i} \int_{\Gamma_{k}}(z-T)^{-1} d z
$$

is the projection of $\mathcal{K}$ onto the subspace

$$
K_{k}=\left\{F \in \mathcal{K}:\left(T-\lambda_{k}\right)^{m_{k}} F=b_{k}^{m_{k}}(T) F=0\right\}
$$

along its complementary space $\mathcal{K}_{\hat{k}}$, where $\mathcal{K}_{k}, \mathcal{K}_{\hat{k}}$ are $T$-invariant subspaces such that

$$
\mathcal{K}=\mathcal{K}_{k} \oplus \mathcal{K}_{k}^{\wedge}, \quad \sigma\left(T \mid \mathcal{K}_{k}\right)=\left\{\lambda_{k}\right\}, \quad \sigma\left(T \mid \mathcal{K}_{k}^{\prime}\right)=\sigma(T) \backslash\left\{\lambda_{k}\right\}
$$

( $\oplus$ denotes an algebraic direct sum, which is not orthogonal, in general).

Moreover, $P_{k}(T) T=T P_{k}(T)$ (cf. [6, Chapter XI]).

Let $F$ be a cyclic vector for $T$; then, from $\mathcal{K}=\bigvee_{n=0}^{\infty} T^{n} F$, we infer that

$$
K_{k}=P_{k}(T) \mathcal{K}=\bigvee_{n=0}^{\infty} P_{k}(T) T^{n} F=\bigvee_{n=0}^{\infty} T^{n} P_{k}(T) F=\bigvee_{n=0}^{\infty} T_{k}^{n} P_{k}(T) F
$$

Now observe that $\mathbb{M}_{k}=\mathcal{K}_{k}^{1}=B_{k} H_{K}^{2} \in(\mathrm{IN})_{B}^{\tilde{B}}$ and its mif is equal to $b_{k}^{m}$. Since $F_{k}=P_{k}(T) F$ is a cyclic vector for $T_{k}$, we only need to show that $\mathbb{M}_{k} \epsilon$ 
(det) $)_{B}^{\sim}$ and $\operatorname{det} B_{k}=b_{k}^{m_{k}}$, from which the result follows (in fact, using results in

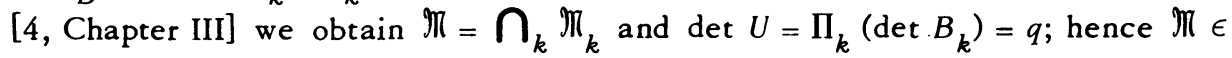
$(\mathrm{det})_{B}^{\sim}$ ).

We have: (1) $\mathcal{K}_{k}=\bigvee_{n=0}^{\infty} T_{k}^{n} F_{k}$, and (2) $\mathcal{K}_{k}$ admits the expression (3.1) of Lemma 3.1. Furthermore, $b_{k}^{m_{k}}\left(T_{k}\right)=0$, and therefore, $T_{k}$ satisfies a polynomial equation of degree $m_{k}:\left(T_{k}^{k}-\lambda_{k}\right)^{m_{k}}=0$. Hence, $\mathcal{K}_{k}=\bigvee_{n=0}^{m_{k}-1} T_{k}^{n} F_{k}$ and $\operatorname{dim} K_{k}$ $\leq m_{k}$.

Now we can apply a result from [4, Chapter III-2]. If $\operatorname{dim} K_{k}<m_{k}$, then the mif of $\mathbb{M}_{k}$ is a Blaschke product of degree $N<m_{k}$, which contradicts our previous result. Hence, $\operatorname{dim} K_{k}=m_{k}$, and we conclude that $B_{k} \in(\mathrm{det})_{B}$ and $m_{k}=$ degree $\left(\operatorname{det} B_{k}\right)=\operatorname{dim} K_{k} \geq \operatorname{degree}\left(\right.$ mif of $\left.B_{k}\right)=m_{k}$; i.e., det $B_{k}=$ mif of $B_{k}=b_{k}^{m} k$.

The following theorem is contained in [10, Theorem 2]:

The ore m 3.3. Let $T \in C_{0}(N)$ (i.e., $T$ can be represented in the form (0.1) where $K$ is a Hilbert space of dimension $N<\infty)$; then the following conditions are equivalent:

(i) there exists a cyclic vector for $T$;

(ii) if $\mathbb{M}=U H_{K}^{2}$ ( $U$ an inner function-operator), then $\operatorname{det} U(z)=q(z), q=$ mif of $U$;

(iii) for each inner factor $p$ of $q$ there exists a unique $T$-invariant subspace $K_{p}$ such that $m_{T \mid K_{p}}=p$, and this subspace has the expression

$$
K_{p}=\{F \in K: p(T) F=0\}
$$

(iv) there is no proper $T$-invariant subspace $\mathcal{L}$ sucb that $m_{T \mid \mathfrak{P}}=q$.

Theorem 3.3'. Let $T \in C_{0}$ be the contraction associated to the IN-subspace M, and let (i),..., (iv) be as in Theorem 3.3. Then:

(1) in the general case (ii) $\Rightarrow$ (iii) $\Rightarrow$ (iv) $\Rightarrow$ (i);

(2) moreover, if $\mathbb{M} \in(\mathrm{IN})_{B}$, then the four properties are equivalent.

Sketch of the proof. (1) (ii) $\Rightarrow$ (iii) and (iv) $\Rightarrow$ (i) follow from Proposition 1.7, Theorem 2.1, and the corresponding argument in [10, Theorem 2]. For the proof that (iii) $\Rightarrow$ (iv), see [10, Theorem 2].

(2) (iv) $\Rightarrow$ (i) is the statement of Corollary 3.2. Hence if $\mathbb{M} \in($ IN) $\underset{B}{ }$, then (i), (ii), (iii) and (iv) are equivalent.

Remark. A closer analys is of the subspaces $\Re_{k}^{\prime \prime}$ of Theorem 2.1, (iii) shows that, if $\mathbb{M} \in(\mathrm{IN})_{B}$ and $T$ has a cyclic vector, then

$$
\mathcal{E}(\mathbb{N})=\bigcup_{k}\left\{g_{\lambda_{k}} \phi_{k}\right\}^{\perp} \text { and } \mathcal{K}_{\sigma}=\bigcup_{k}\left[\mathcal{K} \cap\left\{g_{\lambda_{k}} \phi_{k}\right\}^{\perp}\right]
$$

for some unitary vectors $\phi_{k} \in K$; i.e., $\mathcal{E}(\pi)$ and $\mathcal{K}_{\sigma}$ are countable unions of subspaces of codimension one. 
Corollary 3.4. If $\mathbb{M} \in(\mathrm{IN})_{B}^{-}$, then the restriction of $T$ to any cyclic $T$ invariant subspace is a weak contraction.

Corollary 3.5. If $\mathbb{M}_{=} \mathbb{M}_{b} \cap \mathbb{M}_{d} \cap \mathbb{M}_{s}$ is the decomposition of the IN-subspace $\pi$ with mif $q=b d s$, given by Proposition 1.5 , and $T$ admits a cyclic vector, then $\pi_{b}=B H_{K}^{2} \in(\mathrm{det})_{B}^{-}$and $\operatorname{det} B=b=$ mif of $B$.

2. Theorem 3.6. Let $\mathbb{M}=B H_{K}^{2} \in(\mathrm{IN})_{B}^{\sim}$ with mif $q=\Pi_{k} b_{k}^{m_{k}}$ and let $\mathcal{K}=$ $\bigvee_{k} K_{k}$ be the decomposition of $\mathcal{K}=\pi^{\perp}$ associated to the zeroes of $q$ (i.e., the one given by (3.2) to (3.4)). Let $T$ be defined as usual; then

(i) if $\operatorname{dim} K_{k}=t_{k}<\infty$, for all $k$, then $T$ admits a (unique) Jordan model; i.e., there exists a Jordan operator $J$ such that $T>J>T$. Moreover, if $J=$ $J\left(q_{1}, q_{2}, \ldots, q_{n}, \ldots\right)$, then $q_{1}=q$ and length $J=\mu_{T}$.

(ii) Moreover, if $M \in(\mathrm{det})_{B}^{-}$, then the IN-subspace 'M' associated to $J$ belongs to $(\mathrm{det})_{B}$ and

$$
b=\operatorname{det} B=\prod_{k} b_{k}^{t} k=\prod_{k} q_{n} .
$$

(iii) Conversely, if $T>J$ (or $J>T$ ), where $J$ is a Jordan operator such that $\Pi_{n} q_{n}(z)$ converges (uniformly on compact subsets of $D$ ) to a function $b(z$ ) $\not 0$, then $J$ is the Jordan model of $T, \mathbb{M} \in(\mathrm{det})_{B}$ and the equalities (3.5) are satisfied.

Observe that the proof of [11, Proposition 3] can be easily modified to show that

Proposition 3.7. Let

$$
J=J\left(q_{1}, q_{2}, \ldots, q_{n}, \ldots\right) \text { and } J^{\prime}=J^{\prime}\left(q_{1}^{\prime}, q_{2}^{\prime}, \ldots, q_{1}^{\prime}, \ldots\right)
$$

be two Jordan operators of lengths $\omega$ and $\omega^{\prime}$ resp., and assume that $J>J$ '. Then $\omega=\omega^{\prime}, q_{n}^{\prime}=q_{n}$ (for all $n$ ), and botb operators coincide (up to unitary equivalence).

Proof of Theorem 3.6. Let $K=\bigvee_{k} K_{k}$ and let $T_{k}=P_{k}(T) T=T P_{k}(T)$ as in Corollary 3.2. Then $\mu_{T_{k}} \leq \mu_{T}$, for all $k$. Since $T_{k}$ satisfies a polynomial equation of degree $m_{k}$, it is clear that $\mu_{T_{k}}<\infty$ if and only if $\operatorname{dim} K_{k}<\infty$.

(i) Let $\operatorname{dim} K_{k}=t_{k}<\infty$, for all $k$. Then (see [11, Theorem 2]) there exists a Jordan operator of finite length

$$
J_{k}^{\prime}=J\left(b_{k}^{m_{k}}, \cdots, b_{k}^{m_{k}}, b_{k}^{m_{k}-1}, \ldots, b_{k}^{m_{k}-1}, \cdots, b_{k}, \cdots, b_{k}\right),
$$

where $r_{m_{k}-1}$ terms are equal to $b_{k}^{m_{k}}, r_{m_{k}-2}-r_{m_{k}-1}$ terms are equal to $b_{k}^{m_{k}-1}$, $\cdots, r_{0}-r_{1}$ terms are equal to $b_{k}$,

$$
t_{k}=\sum_{b=0}^{m_{k}-1}(b+1)\left(r_{n}-r_{b+1}\right) \quad\left(r_{m_{k}}=0\right)
$$

and $J_{k}^{\prime}$ is similar to $T_{k}$. 
Define the operator $J$ as follows: $J=J\left(q_{1}, q_{2}, \ldots, q_{n}, \ldots\right)$, where $q_{n}=$ $\Pi_{k}\left(n\right.$th term of $J_{k}^{\prime}$ ) (the product is taken over all those $k$ such that the length of $J_{k}^{\prime}$ is $\geq n$ ).

Claim. $J$ is a Jordan model for $T$. Let $\mathcal{K}^{\prime}$ be the space on which $J$ acts and let $K^{\prime}=\bigvee_{k} K_{k}^{\prime}, K^{\prime}=K_{k}^{\prime} \oplus K_{k}^{\prime} \hat{,}, P_{k}^{\prime}(J)$ and $J_{k}=J P_{k}^{\prime}(J)=P_{k}^{\prime}(J) J$ be defined by the formulas (3.2) to (3.4) of Corollary 3.2. Then $J_{k}$ is unitarily equivalent to $J_{k}^{\prime}$; hence it is similar to $T_{k}$. Let $X_{k}: K_{k}^{\prime} \rightarrow \mathcal{K}_{k}$ be an invertible map such that $T_{k} X_{k}=X_{k} J_{k}(k=1,2, \ldots)$ and define the bounded linear map $X: K^{\prime} \rightarrow \mathcal{K}$ by means of $X=\Sigma_{k} c_{k} X_{k} P_{k}^{\prime}(J)\left(c_{k}>0\right.$, for all $\left.k\right)$, where the constants $c_{k}$ are chosen so that $\|X\| \leq 1$ (e.g., we can take $c_{k}=2^{-k}\left\|X_{k}\right\|^{-1}$, in which case $X$ is a compact operator). We want to show that $X$ is actually a quasi-invertible operator from $K^{\prime}$ to $K$, and

$$
T X=X J
$$

Let $\mathfrak{L}_{N}=\bigoplus_{k+1}^{N} K_{k}, \Re_{N}=\bigvee_{k=N+1}^{\infty} K_{k}, P_{\mathfrak{S}_{N}}=\Sigma_{k=1}^{N} P_{k}(T)$ and let $\mathfrak{S}_{N}^{\prime}, \mathfrak{R}_{N}^{\prime}$ and $P_{\mathscr{P}_{N}^{\prime}}$ be similarly defined.

Let $F^{\prime}=F_{1}^{\prime}+F_{2}^{\prime}+\cdots+F_{N}^{\prime} \in \mathfrak{L}_{N}^{\prime}\left(F_{k}^{\prime} \in \mathcal{K}_{k}^{\prime}\right)$. Then

$$
\begin{aligned}
X J F^{\prime} & =\sum_{k=1}^{N} X\left(J F_{k}^{\prime}\right)=\sum_{k=1}^{N} c_{k} X_{k} J F_{k}^{\prime}=\sum_{k=1}^{N} c_{k} T X_{k} F_{k}^{\prime} \\
& =T\left(\sum_{k=1}^{N} c_{k} X_{k} F_{k}^{\prime}\right)=T X F^{\prime} .
\end{aligned}
$$

Since $\bigcup_{N} \mathscr{Q}_{N}^{\prime}$ is dense in $K^{\prime}$ and $X$ is continuous, we obtain (3.6). Moreover, it is not hard to infer from the definition of $X$ that $X\left(\mathscr{Q}_{N}^{\prime}\right)=\mathscr{L}_{N}$ and $X \mid \mathfrak{S}_{N}^{\prime}: \mathfrak{Q}_{N}^{\prime} \rightarrow \mathfrak{L}_{N}$ is an invertible map, for all $N$. Therefore,

$$
\overline{X\left(\mathcal{K}^{\prime}\right)}=\overline{X\left(\bigcup_{N} \mathfrak{Q}_{N}^{\prime}\right)}=\overline{\bigcup_{N} X\left(\mathscr{Q}_{N}^{\prime}\right)}=\overline{\bigcup_{N} \mathfrak{L}_{N}}=\mathcal{K} \text {. }
$$

It only remains to show that $\operatorname{ker}(X)=\{0\}$.

Observe that $\mathfrak{Q}_{N}$ and $\Re_{N}$ form a positive angle in $\mathcal{K}$, and $\mathcal{K}=\mathscr{L}_{N} \oplus \Re_{N}$; moreover, $\mathcal{Q}_{N}^{\prime}$ and $\Re_{N}^{\prime}$ have similar properties in $K^{\prime}$.

Let $G^{\prime} \in \operatorname{ker}(X)$. Then $G^{\prime}$ has a unique decomposition $G^{\prime}=G_{N}^{\prime}+F_{N}^{\prime}$, where $G_{N}^{\prime} \in \mathscr{Q}_{N}^{\prime}$ and $F_{N}^{\prime} \in \Re_{N}^{\prime}$.

Then $G_{N}=X\left(G_{N}^{\prime}\right) \in \mathscr{L}_{N}$ and $F_{N}=X\left(F_{N}^{\prime}\right) \in R_{N}$. Since $X\left(G^{\prime}\right)=0$, we have: $G_{N}=-F_{N} \in \mathscr{L}_{N} \cap \mathscr{R}_{N}=\{0\}$.

On the other hand, the fact that $X \mid \mathscr{\complement}_{N}^{\prime}$ is invertible implies that $G_{N}^{\prime}=0$, for all $N$. Hence

$$
\operatorname{ker}(X) \subset \bigcap_{k} R_{N}^{\prime}=\{0\}
$$


Therefore, $X$ is quasi-invertible. Thus we have: $T>J$.

The same argument proves that $Y=\Sigma_{k} c_{k}^{\prime} X_{k}^{-1} P_{k}(T)$ is a quasi-invertible and $J Y=Y T$; i.e., $J>T>J$. Hence $J$ is a Jordan model for $T$, and $J^{*}>T^{*}$ $>J^{*}$ (i.e., $J^{*}$ is a Jordan model for $T^{*}$ ).

Now, the uniqueness of $J$ (and $J^{*}$ ) follows from Proposition 3.4. Thus we have $J^{*}=J\left(q_{1}, q_{2}, \ldots, q_{n}, \ldots\right)$, where $q^{\sim}(z)=\overline{q(\bar{z})}$; in particular, $\mu_{T^{*}}=\mu_{T}=$ length of $J=$ length of $J^{*}$.

(ii) If $K_{k}^{\perp}=\mathbb{M}_{k}=B_{k} H_{K}^{2}, K_{k}^{\prime \perp}=\mathbb{M}_{k}^{\prime}=B_{k}^{\prime} H_{K}^{2}$, and $\mathbb{M} \in(\mathrm{det})_{B}^{-}$, then it follows from the constructions of (i) that

$$
b=\operatorname{det} B=\prod_{k} \operatorname{det} B_{k}=\prod_{k} \operatorname{det} B_{k}^{\prime}=\prod_{k} b_{k}^{t_{k}}=\prod_{n} q_{n} .
$$

(iii) If $T>J$, then $\operatorname{dim} K_{k}=\operatorname{dim} K_{k}^{\prime}=t_{k}$, for all $k$.

If $t_{k}$ is not finite for some $k$, then $b_{k}^{m}$ divides $\Pi_{n} q_{n}$ for all values of $m$, and this implies $b(z) \equiv 0$, contradicting our assumption. Hence $t_{k}$ is always finite.

It is clear that $b_{k}^{t}{ }^{k}$ divides $b$ for all $k$; thus $\Pi_{k} b_{k}^{t} k b=\Pi_{n} q_{n}\left(\Pi_{k} b_{k}^{t} k\right.$ converges and it defines an inner function) and therefore $\mathbb{M} \in(\mathrm{det})_{B}^{\sim}$.

Now (iii) follows from (i) and (ii).

As in [11, Corollaries 1 and 2], we have

Corollary 3.8. Let $\mathbb{M}=B H_{K}^{2} \in(\mathrm{IN})_{B}^{\sim}, \mathbb{M}^{\prime}=B^{\prime} H_{K}^{2} \in \mathcal{F}^{\sim}$ and assume that $\mathcal{K}=$ $\mathbb{M}^{\perp}=\bigvee_{k} \mathcal{K}_{k}$, where $\sigma\left(T \mid \mathcal{K}_{k}\right)=\left\{\lambda_{k}\right\}$ and $\operatorname{dim} \mathcal{K}_{k}=t_{k}<\infty$. Then

(i) If $T>T^{\prime}$ or $T^{\prime}>T$, then $T$ and $T^{\prime}$ bave the same Jordan model. Conversely, if $T$ and $T^{\prime}$ bave the same Jordan model, then they are quas $i$ similar. If, in particular, $\mathbb{M} \in(\mathrm{det})_{B^{2}}$, then $\mathbb{M}^{\prime} \in(\mathrm{det})_{B}$ and $\operatorname{det} B^{\prime}=\operatorname{det} B$.

(ii) $T$ and its restriction $T^{\prime \prime}$ to a proper $T$-invariant subspace cannot bave the same Jordan model.

Proof. If $T \succ T^{\prime}$ or $T^{\prime}>T$, then $\mathbb{M}^{\prime} \in(\mathrm{IN})_{B}^{\sim}$ and it has the same mif as M ([9, Proposition VII, 2.1]). Let $\mathcal{K}=\bigvee_{k} \mathcal{K}_{k}, \mathcal{K}^{\prime}=\bigvee_{k} K_{k}^{\prime}$ be the decomposition (3.2) to (3.4). It follows from the proof of Theorem 3.6 that $\operatorname{dim} K_{k}^{\prime}=\operatorname{dim} K_{k}=t_{k}$, for all $k$, and the proof proceeds along the lines of Theorem 3.6.

3. The sufficient condition for the existence of a Jordan model for the contraction $T$ (where $\mathbb{M} \in(\mathrm{IN})_{B}^{\sim}$ ) is clearly not necessary; however, we believe that the only interesting cases are those considered in Theorem 3.6. In fact, the operators satisfying the condition (i) of Theorem 3.6 can be redefined as those $C_{0}$-contractions such that $I-T^{*} T$ is compact and the associated invariant subspace $M$ belongs to the subclass (IN) ${ }_{B}$; this definition (and the one corresponding to the determinant class, or some intermediate class) can be naturally extended. 
We conjecture that Theorems 3.3 and 3.6 (and related results) remain true without the assumption that the mif of the IN-subspace $\mathbb{M}$ is a Blaschke product; moreover, this seems to be the most general situation in which all results of this type are valid.

For example, if $\mathbb{N}=z H_{K}^{2}$, where $K$ is an infinite dimensional Hilbert space, then $\mathcal{K}=K$ and $T=0$; then $T$ and its restriction to any infinite dimensional subspace have the same Jordan model; namely, $J=J(z, z, z, \ldots)$. This proves that the hypothesis of Corollary 3.8, (ii) cannot be relaxed.

A final remark. It was proved in [11] that if $T \in C_{0}(N)$, then it has a Jordan model of length $\omega \leq N$.

Assume that $\mathbb{M}=U H_{K}^{2} \in(\mathrm{det})^{\tilde{w}}$ with mif $q$ and $\operatorname{det} U=p$ and assume that $T$ admits a Jordan model of finite length $J\left(q_{1}, q_{2}, \ldots, q_{\omega}\right)$, where $q_{1}=q$ and $p=$ $\Pi_{n=1}^{\omega} q_{n} ;$ then $q^{\omega}<\Pi_{n=1}^{\omega} q_{n}=p$, and therefore,

$$
\omega \geq \inf \left\{k: q^{k}<p\right\}=\omega_{0} .
$$

Let $b=\Pi_{k=1}^{\infty} b_{k}^{k}, r=\Pi_{k=1}^{\infty} b_{k}$ be two Blaschke products with the same zeroes (all the zeroes of $r$ have order one), and let

$$
J=J\left(\prod_{k=1}^{\infty} b_{k}, \prod_{k=2}^{\infty} b_{k}, \cdots, \prod_{k=n}^{\infty} b_{k}, \cdots\right) \text { and } J^{\prime}=J(r) \oplus J .
$$

In the first case, there is no finite $\omega_{0}$ satisfying the above condition; in the second one, $\omega_{0}=2$. However, $J^{\prime}$ has also infinite length.

Note. After this paper was written, the author received the preprint "Boundedness from measure theory", by Henry Helson. In this article, H. Helson gives (among other results) new proofs of the constant directions theorem of [8] and several results related to $\$ 2$.

\section{REFERENCES}

1. Ju. P. Ginzburg, On the multiplicative representation of bounded analytic operator-functions, Dokl. Akad. Nauk SSSR 170 (1966), 23-26 = Soviet Math. Dokl. 7 (1966), 1125-1128. MR 34 \#611.

2. - Multiplicative representations of operator-functions of bounded characteristic, Uspehi Mat. Nauk 22 (1967), no. 1, (133), 163-165. (Russian) MR 34 \#6511.

3. H. Helson, Lectures on invariant subspaces, Academic Press, New York and London, 1964. MR $30 \# 1409$.

4. D. A. Herrero, Inner function-operators, Thesis, University of Chicago, Chicago III., 1970.

5. K. Hoffman, Banach spaces of analytic functions, 2nd. ed., Prentice-Hall, Englewood Cliffs, 1965.

6. F. Riesz and B. Sz.-Nagy, Functional analysis, Akad. Kiadó, Budapest, 1953; English transl., Ungar, New York, 1955. MR 15, 132; MR 17, 175.

7. M. J. Sherman, Invariant subspaces containing all analytic directions, J. Functional Analysis 3 (1969), 164-172.

8. Invariant subspaces containing all constant directions, J. Functional Analysis 8 (1971), 82-85. 
9. B. Sz.-Nagy and C. Foias, Analyse harmonique des opérateurs de l'espace de Hilbert, Masson, Paris, A kad. Kiadó, Budapest, 1967. MR 37 \#778.

10. - Opérateurs sans multiplicité, Acta Sci. Math. (Szeged) 30 (1969), 1-18.

11. Modèle de Jordan pour une classe d'opérateurs de l'espace de Hilbert, Acta Sci. Math. (Szeged) 31 (1970), 91-115. MR 41 \#9038.

12. - Local characterization of operators of class $C_{0}, \mathrm{~J}$. Functional A nalys is 8 (1971), 76-81.

13. Compléments à l'étude des opérateurs de closse $C_{0}$, Acta Sci. Math. (Szeged) 31 (1970), 287-296.

DEPARTMENT OF MATHEMATICS, UNIVERSITY OF CHICAGO, CHICAGO, ILLINOIS 60637

DEPARTMENT OF MATHEMATICS, STATE UNIVERSITY OF NEW YORK, ALBANY, NEW YORK 12222

Current address: Universidade Estadual de Campinas, I.M.E.C.C., Caixa Postal 1170, 13100 Campinas, São Paulo, Brasil 